\title{
Analytical solution of thermally developing microtube heat transfer including axial conduction, viscous dissipation, and rarefaction effects is
}

\author{
Murat Barışık $^{\mathrm{a}, *}$, Almıla Güvenç Yazıcıoğlu ${ }^{\mathrm{b}}$, Barbaros Çetin ${ }^{\mathrm{c}}$, Sadık Kakaç ${ }^{\mathrm{d}}$ \\ a Izmir Institute of Technology, Mechanical Engineering Department, Izmir 35430, Turkey \\ b Middle East Technical University, Mechanical Engineering Department, Ankara 06800, Turkey \\ c Bilkent University, Mechanical Engineering Department, Ankara 06800, Turkey \\ d TOBB Economics and Technology University, Mechanical Engineering Department, Ankara 06560, Turkey
}

\section{A R T I C L E I N F O}

Available online 16 May 2015

\section{Keywords:}

Extended Graetz problem

Micropipe heat transfer

Slip flow

Rarefaction effect

Axial conduction

Viscous dissipation

\begin{abstract}
A B S T R A C T
The solution of extended Graetz problem for micro-scale gas flows is performed by coupling of rarefaction, axial conduction and viscous dissipation at slip flow regime. The analytical coupling achieved by using Gram-Schmidt orthogonalization technique provides interrelated appearance of corresponding effects through the variation of non-dimensional numbers. The developing temperature field is determined by solving the energy equation locally together with the fully developed flow profile. Analytical solutions of local temperature distribution, and local and fully developed Nusselt number are obtained in terms of dimensionless parameters: Peclet number, Knudsen number, Brinkman number, and the parameter Kappa accounting temperature-jump. The results indicate that the Nusselt number decreases with increasing Knudsen number as a result of the increase of temperature jump at the wall. For low Peclet number values, temperature gradients and the resulting temperature jump at the pipe wall cause Knudsen number to develop higher effect on flow. Axial conduction should not be neglected for Peclet number values less than 100 for all cases without viscous dissipation, and for short pipes with viscous dissipation. The effect of viscous heating should be considered even for small Brinkman number values with large length over diameter ratios. For a fixed Kappa value, the deviation from continuum increases with increasing rarefaction, and Nusselt number values decrease with an increase in Knudsen number.
\end{abstract}

(C) 2015 Published by Elsevier Ltd.

\section{Introduction}

Interest in micro- and nanoscale heat transfer has been explosively increasing in accordance with the developments in MEMS and nanotechnology during the last two decades. The aim of cooling micro- and nanoscale devices is an important subject for most engineering applications. Cooling of devices having the dimensions of microns is a completely different problem than what is analyzed in the macro world which makes investigation of the flow characteristics of microand nanoscale flows a key research field.

One can understand some of the advantages of using micro- and nanoscale devices in heat transfer, starting from the single phase internal flow correlation for convective heat transfer,

$h=\frac{N u \cdot k}{D}$

where $h$ is the convective heat transfer coefficient, $N u$ is the Nusselt number, $k$ is the thermal conductivity of the fluid and $D$ is the hydraulic

\footnotetext{
is Communicated by W.J. Minkowycz.

* Corresponding author

E-mail address: muratbarisik@iyte.edu.tr (M. Barışık).
}

diameter of the channel or duct. In internal fully developed laminar flows, Nu becomes a constant. Theory calculates $\mathrm{Nu}=3.657$ for the constant wall temperature case, and $\mathrm{Nu}=4.364$ for the constant heat flux case [1]. As Reynolds number ( $\mathrm{Re}$ ) is proportional to hydraulic diameter, fluid flow in channels of small hydraulic diameter will predominantly be laminar. The above correlation therefore indicates that the heat transfer coefficient increases as channel diameter decreases. As a result of the hydraulic diameter being order of tens or hundreds of micrometers in forced convection microscale applications, heat transfer coefficient should be extremely high. However, the question is whether the earlier mentioned theoretical $\mathrm{Nu}$ values are still the same for micro flows. While the system size is decreased to increase the surface to volume ratio and enhance the heat transfer, probable effects of micro-level small size onto transport characteristics should be carefully examined.

In a macroscale, continuum approach is the basis for most of the cases. However, continuum hypothesis may not be applicable for some of the micro-scale fluid transport and heat transfer problems, especially for micro gas flows. While the ratio of the average distance traveled by the molecules without colliding with each other, the mean free path $(\lambda)$, to the characteristic length of the flow $(L)$ is increases, the continuum approach fails to be valid, and the fluid modeling shifts from continuum model to molecular model. This ratio is known as Knudsen number 


\begin{tabular}{|c|c|}
\hline \multicolumn{2}{|c|}{ Nomenclature } \\
\hline$B r$ & Brinkman number \\
\hline$C_{1}$ & coefficient in Eq. (11d) \\
\hline$c_{p}$ & constant pressure specific heat, $\mathrm{J} / \mathrm{kgK}$ \\
\hline$D$ & tube diameter, $\mathrm{m}$ \\
\hline$F_{m}$ & tangential momentum accommodation coefficient \\
\hline$F_{t}$ & thermal accommodation coefficient \\
\hline$h$ & convective heat transfer coefficient, $\mathrm{W} / \mathrm{m}^{2} \mathrm{~K}$ \\
\hline$K n$ & Knudsen number, $\lambda / L$ \\
\hline$k$ & thermal conductivity, $\mathrm{W} / \mathrm{mK}$ \\
\hline $\mathrm{Nu}$ & Nusselt number \\
\hline $\mathrm{Pe}$ & Peclet number, $\mathrm{k} / \rho C_{\mathrm{p}}$ \\
\hline $\operatorname{Pr}$ & Prandtl number, $v / \alpha$ \\
\hline$R$ & tube radius, $\mathrm{m}$ \\
\hline$r$ & radial coordinate \\
\hline$r^{*}$ & dimensionless radial coordinate \\
\hline$T$ & fluid temperature, $\mathrm{K}$ \\
\hline$u$ & velocity, m/s \\
\hline$u^{*}$ & dimensionless velocity \\
\hline$x$ & axial coordinate \\
\hline$x^{*}$ & dimensionless axial coordinate, $\mathrm{x} /(\mathrm{R} \mathrm{Pe})$ \\
\hline \multicolumn{2}{|c|}{ Greek symbols } \\
\hline$\alpha$ & thermal diffusivity, $\mathrm{m}^{2} / \mathrm{s}$ \\
\hline$\gamma$ & specific heat ratio \\
\hline$\lambda$ & mean free path, $\mathrm{m}$ \\
\hline$\lambda_{n}$ & eigenvalue \\
\hline$\mu$ & dynamic viscosity, $\mathrm{kg} / \mathrm{ms}$ \\
\hline$\kappa$ & coefficient in Eq. (11d) \\
\hline$\nu$ & kinematic viscosity, $\mathrm{m}^{2} / \mathrm{s}$ \\
\hline$\Theta$ & dimensionless temperature, $\left(\mathrm{T}-\mathrm{T}_{\mathrm{w}}\right) /\left(\mathrm{T}_{\mathrm{i}}-\mathrm{T}_{\mathrm{w}}\right)$ \\
\hline$\eta$ & dimensionless radial coordinate, $\rho_{s} r / R$ \\
\hline$\xi$ & dimensionless axial coordinate, $\rho_{s}^{2}\left(2-\rho_{s}^{2}\right)$ x / (R Pe) \\
\hline$\xi^{*}$ & dimensionless axial coordinate, $\rho_{\mathrm{s}}^{2}\left(2-\rho_{\mathrm{s}}^{2}\right) \mathrm{x} /(\mathrm{R})$ \\
\hline \multicolumn{2}{|c|}{ Subscripts } \\
\hline$i$ & inlet \\
\hline$s$ & slip \\
\hline$w$ & wall \\
\hline
\end{tabular}

$\mathrm{Kn}=\lambda / L$

which is employed to determine the flow characteristics [2]. The flow is considered as continuum flow for small values of $\mathrm{Kn}(<0.01)$, and the well known Navier-Stokes equations together with the no-slip and no-temperature jump boundary condition are applicable for the flow
Table 1

The first 20 eigenvalues and corresponding coefficients for $\mathrm{Pe}=10,5$, and 1 .

\begin{tabular}{|c|c|c|c|c|c|c|}
\hline \multirow[b]{2}{*}{$\mathrm{n}$} & \multicolumn{2}{|l|}{$\mathrm{Pe}=1$} & \multicolumn{2}{|l|}{$\mathrm{Pe}=5$} & \multicolumn{2}{|l|}{$\mathrm{Pe}=10$} \\
\hline & $\lambda_{\mathrm{n}}$ & $A_{n}$ & $\lambda_{n}$ & $A_{n}$ & $\lambda_{n}$ & $A_{n}$ \\
\hline 1 & 1.4298 & 1.6059 & 2.3853 & 1.5774 & 2.5969 & 1.5354 \\
\hline 2 & 2.2776 & -1.0736 & 4.5109 & -1.0458 & 5.5469 & -0.9861 \\
\hline 3 & 2.8850 & 0.8589 & 5.9765 & 0.8447 & 7.7139 & 0.7795 \\
\hline 4 & 3.3855 & -0.7357 & 7.1579 & -0.7341 & 9.4592 & -0.6875 \\
\hline 5 & 3.8211 & 0.6534 & 8.1744 & 0.6578 & 10.9532 & 0.6309 \\
\hline 6 & 4.2119 & -0.5935 & 9.0798 & -0.6001 & 12.2780 & -0.5865 \\
\hline 7 & 4.5695 & 0.5475 & 9.9040 & 0.5546 & 13.4796 & 0.5487 \\
\hline 8 & 4.9012 & -0.5107 & 10.6653 & -0.5177 & 14.5862 & -0.5162 \\
\hline 9 & 5.2117 & 0.4804 & 11.3763 & 0.4870 & 15.6171 & 0.4879 \\
\hline 10 & 5.5048 & -0.4549 & 12.0457 & -0.4611 & 16.5856 & -0.4633 \\
\hline 11 & 5.7831 & 0.4331 & 12.6800 & 0.4388 & 17.5017 & 0.4418 \\
\hline 12 & 6.0486 & -0.4142 & 13.2842 & -0.4194 & 18.3731 & -0.4227 \\
\hline 13 & 6.3029 & 0.3975 & 13.8621 & 0.4023 & 19.2055 & 0.4058 \\
\hline 14 & 6.5474 & -0.3827 & 14.4171 & -0.3871 & 20.0039 & -0.3906 \\
\hline 15 & 6.7830 & 0.3694 & 14.9515 & 0.3735 & 20.7719 & 0.3770 \\
\hline 16 & 7.0107 & -0.3574 & 15.4675 & -0.3612 & 21.5128 & -0.3646 \\
\hline 17 & 7.2313 & 0.3465 & 15.9669 & 0.3501 & 22.2292 & 0.3533 \\
\hline 18 & 7.4454 & -0.3366 & 16.4511 & -0.3399 & 22.9235 & -0.3430 \\
\hline 19 & 7.6534 & 0.3274 & 16.9216 & 0.3305 & 23.5975 & 0.3336 \\
\hline 20 & 7.8560 & -0.3190 & 17.3793 & -0.3219 & 24.2528 & -0.3248 \\
\hline
\end{tabular}

field. For $0.01<\mathrm{Kn}<0.1$ flow is in slip-flow regime (slightly rarefied). For $0.1<\mathrm{Kn}<10$ flow is in transition regime (moderately rarefied). Finally, the flow is considered as free-molecular flow for large values of Kn (>10) (highly rarefied); the tool for dealing with this type flow is kinetic theory of gases, Direct Simulation of Monte Carlo (DSMC) [3] and Molecular Dynamics [4-8].

As the characteristic length of the system decreases, the effect of rarefaction comes into picture. Classical no-slip velocity and notemperature jump boundary conditions are not valid for a rarefied fluid flow at micro/nanoscale. Since the fluid particles adjacent to the boundary surface are not in thermodynamic equilibrium with the wall, there would be slip velocity and temperature jump at the channel wall (For a more detailed discussion on these, the readers are referred to the textbook by Gad-el-Hak [9]). For the slip flow regime $(0.01<\mathrm{Kn}<0.1)$, slip-velocity and temperature-jump boundary conditions for a microtube can be defined as follows [2],

$u_{s}=-\frac{2-\sigma_{m}}{\sigma_{m}} \lambda\left(\frac{d u}{d r}\right)_{r=R}$

$T-T_{s}=-\frac{2-\sigma_{t}}{\sigma_{t}} \frac{2 \gamma}{\gamma+1} \frac{\lambda}{\operatorname{Pr}}\left(\frac{\partial T}{\partial r}\right)_{r=R}$

In these equations, $\sigma_{m}$ is the tangential momentum accommodation coefficient, $\sigma_{t}$ is the thermal accommodation coefficient, and $\gamma$ is the specific heat ratio. These slip flow models are successfully employed

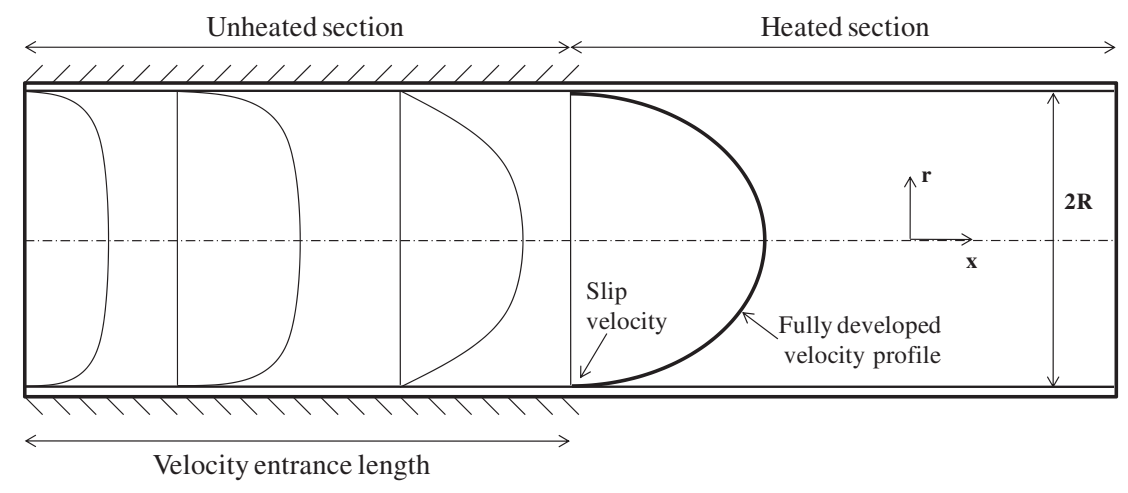

Fig. 1. Geometry of the problem. 
Table 2

Fully developed Nusselt number with $\mathrm{Kn}=0, \mathrm{Br}=0$ for different Peclet number values.

\begin{tabular}{lllrllrll}
\hline $\mathrm{Pe}$ & $\mathrm{Nu}_{\mathrm{fd}}$ & $\lambda_{1}$ & $\mathrm{Pe}$ & $\mathrm{Nu}_{\mathrm{fd}}$ & $\lambda_{1}$ & $\mathrm{Pe}$ & $\mathrm{Nu}_{\mathrm{fd}}$ & $\lambda_{1}$ \\
\hline $10^{9}$ & 3.65679 & 2.70436 & 300 & 3.65684 & 2.70423 & 20 & 3.66754 & 2.67460 \\
$10^{6}$ & 3.65679 & 2.70436 & 200 & 3.65691 & 2.70406 & 10 & 3.69518 & 2.59693 \\
1000 & 3.65680 & 2.70435 & 100 & 3.65724 & 2.70313 & 8 & 3.71247 & 2.54742 \\
900 & 3.65680 & 2.70435 & 90 & 3.65735 & 2.70284 & 6 & 3.74302 & 2.45812 \\
800 & 3.65680 & 2.70435 & 80 & 3.65749 & 2.70244 & 5 & 3.76729 & 2.38530 \\
700 & 3.65680 & 2.70434 & 70 & 3.65771 & 2.70185 & 4 & 3.80153 & 2.27947 \\
600 & 3.65681 & 2.70433 & 60 & 3.65803 & 2.70094 & 2 & 3.92236 & 1.86754 \\
500 & 3.65681 & 2.70431 & 50 & 3.65858 & 2.69945 & 1 & 4.02735 & 1.42981 \\
400 & 3.65682 & 2.70429 & 40 & 3.65957 & 2.69671 & $10^{-6}$ & 4.18065 & 0.00155
\end{tabular}

to consider the effect of rarefaction on microscale flow [10] while good agreements are obtained with experimental measurements [11].

Most of the existing studies on microscale heat transfer successfully used Eqs. (3) and (4) to consider non-continuum effects developed due to small scale rarefaction. However, additional complications occur at the micron-levels that the effect of viscous dissipation, axial conduction and thermal entrance region should also be considered in the analysis of microscale flows.

Traditionally known as the Graetz problem, thermal entrance region of a tube flow was first investigated by Graetz [12], and later independently by Nusselt [13], analytically. The authors both worked on hydrodynamically developed and thermally developing flow for constant wall temperature boundary condition. There requires, so-called, extension of Graetz problem to include additional effects in order to solve the microscale problems. First, most of the micro-flows have small Peclet number (i.e. Pe $O(1)$ ) due to small Re that the axial conduction cannot be neglected since the convection term no longer dominates the conduction term in the axial direction. Analytical consideration of Gratez problem with axial conduction has been a very interesting problem due to the resulting non-self-adjoint eigenvalue problem [14]. Second, the effect of viscous dissipation on heat transfer becomes significant for flows at microscale since the wall-to-fluid temperature difference is small [11]. There exist multiple studies conducted so far to include effects of rarefaction [15], viscous dissipation, and axial conduction [16] for microscale flows. Hadjiconstantinou and Simek [17] studied the effect of axial conduction for thermally fully developed flows in microchannels. Jeong and Jeong [18] tried to consider streamwise conduction and viscous dissipation in microchannels using numerical procedures. Similarly, Cetin et al. [19-22] performed studies to extend Gratez problem to include micro-scale effects. Dutta et al. [23] and Horiuchi et al. [24] studied the thermal characteristics of mixed electroosmotic and pressure-driven flow with axial conduction analytically where Gram-Schmidt orthogonalization procedure is used to generate orthogonal eigenfunctions. However, there is no report on analytical coupling of all micro-scale complications for the classical constant temperature Gratez problem due to its mathematical difficulties.

In this work, our objective is to couple the rarefaction, viscous dissipation, and axial conduction effects analytically. Mathematical challenge will be eliminated by using Gram-Schmidt orthogonalization accompanied with the Gauss quadrature. Solution for the heat transfer in thermally developing flow inside a microtube in the slip-flow regime will be performed with constant wall temperature. For such case, the energy equation will be solved by using confluent hypergeometric functions in order to provide a fundamental understanding of the effects of the non-dimensional parameters on heat transfer characteristics.

\section{Analysis}

The geometry of the problem considered in this study is shown in Fig. 1. The unheated part is included to ensure the fully developed velocity profile. The coordinate system is placed at the center of the microtube. Fully developed velocity profile inside a microtube is calculated as,

$$
\frac{u}{u_{m}}=\frac{2\left(1-(r / R)^{2}\right)+8 K n}{1+8 K n}
$$

By defining slip radius as suggested by Larrode et al [25],

$$
\rho_{s}^{2}=\frac{1}{1+4 K n}
$$

and a new radial coordinate,

$\eta=\frac{r}{R} \rho_{s}$

the fully developed velocity profile can be rewritten as,

$\frac{u}{u_{m}}=\frac{2}{2-\rho_{s}^{2}}\left(1-\eta^{2}\right)$

which has the same functional as Poiseuille flow.

Energy equation and the boundary conditions for a flow inside a microtube with axial conduction and viscous dissipation can be written as,

$$
\begin{aligned}
& u \frac{\partial T}{\partial x}=\frac{\alpha}{r} \frac{\partial}{\partial r}\left(r \frac{\partial T}{\partial r}\right)+\alpha \frac{\partial^{2} T}{\partial x^{2}}+\frac{\nu}{C_{p}}\left(\frac{\partial u}{\partial r}\right)^{2} \\
& r=0, \quad \frac{\partial T}{\partial r}=0 \\
& r=R, \quad T-T_{w}=-\frac{2-F_{t}}{F_{t}} \frac{2 \gamma}{\gamma+1} \frac{\lambda}{P r}\left(\frac{\partial T}{\partial r}\right)_{r=R}
\end{aligned}
$$

\begin{tabular}{|c|c|c|c|c|c|c|c|c|c|c|c|}
\hline \multicolumn{3}{|c|}{$\mathrm{Pe}=1.0$} & \multicolumn{3}{|c|}{$\mathrm{Pe}=2.0$} & \multicolumn{3}{|c|}{$\mathrm{Pe}=5.0$} & \multicolumn{3}{|c|}{$\mathrm{Pe}=10$} \\
\hline $\mathrm{Nu}_{\mathrm{fd}}$ & $\mathrm{Nu}_{\mathrm{fd}}{ }^{*}$ & $\mathrm{Nu}_{\mathrm{fd}}{ }^{* *}$ & $\mathrm{Nu}_{\mathrm{fd}}$ & $\mathrm{Nu}_{\mathrm{fd}}{ }^{*}$ & $\mathrm{Nu}_{\mathrm{fd}}{ }^{* *}$ & $\mathrm{Nu}_{\mathrm{fd}}$ & $\mathrm{Nu}_{\mathrm{fd}}{ }^{*}$ & $\mathrm{Nu}_{\mathrm{fd}}{ }^{* * *}$ & $\mathrm{Nu}_{\mathrm{fd}}$ & $\mathrm{Nu}_{\mathrm{fd}}{ }^{*}$ & $\mathrm{Nu}_{\mathrm{fd}}{ }^{* *}$ \\
\hline 4.027 & 4.028 & 4.030 & 3.922 & 3.922 & 3.925 & 3.767 & 3.767 & 3.767 & 3.695 & 3.695 & 3.697 \\
\hline
\end{tabular}

$x=0, T=T_{i}$.

Table 3

Comparison of fully developed Nusselt number with $\mathrm{Kn}=0, \mathrm{Br}=0$ for different $\mathrm{Pe} \leq 10$ with literature.

$\mathrm{Nu}_{\mathrm{fd}}$ : results for present study.

$\mathrm{Nu}_{\mathrm{fd}}{ }^{*}$ : results from Cetin et al. [20].

$\mathrm{Nu}_{\mathrm{fd}}{ }^{* *}$ : results from Shah and London [29].

$\mathrm{Nu}_{\mathrm{fd}}{ }^{* * *}$ : results from Lahjomri and Oubarra [27]. 


\section{Table 4}

Comparison of fully developed Nusselt number with $\mathrm{Br}=0$ for different Knudsen number values and $\kappa$ with literature.

\begin{tabular}{|c|c|c|c|c|c|c|c|c|c|c|}
\hline \multirow[t]{2}{*}{ Kn } & \multicolumn{2}{|c|}{$\mathrm{Pe}=1$} & \multicolumn{2}{|c|}{$\mathrm{Pe}=2$} & \multicolumn{2}{|c|}{$\mathrm{Pe}=5$} & \multicolumn{2}{|c|}{$\mathrm{Pe}=10$} & \multicolumn{2}{|c|}{$\mathrm{Pe}=1000$} \\
\hline & $\mathrm{Nu}_{\mathrm{fd}}$ & $\mathrm{Nu}_{\mathrm{fd}}{ }^{*}$ & $\mathrm{Nu}_{\mathrm{fd}}$ & $\mathrm{Nu}_{\mathrm{fd}}{ }^{*}$ & $\mathrm{Nu}_{\mathrm{fd}}$ & $\mathrm{Nu}_{\mathrm{fd}}{ }^{*}$ & $\mathrm{Nu}_{\mathrm{fd}}$ & $\mathrm{Nu}_{\mathrm{fd}}{ }^{*}$ & $\mathrm{Nu}_{\mathrm{fd}}$ & $\mathrm{Nu}_{\mathrm{fd}}{ }^{*}$ \\
\hline 0 & 4.027 & 4.028 & 3.922 & 3.922 & 3.767 & 3.767 & & 3.695 & 3.657 & 3.656 \\
\hline 0.04 & 3.603 & 3.604 & 3.517 & 3.517 & 3.387 & 3.387 & 3.325 & 3.325 & 3.292 & 3.292 \\
\hline 0.08 & 3.093 & 3.093 & 3.035 & 3.036 & 2.949 & 2.949 & 2.908 & 2.909 & 2.886 & 2.887 \\
\hline
\end{tabular}

$\mathrm{Nu}_{\mathrm{fd}}$ : approximate results for the present study.

$\mathrm{Nu}_{\mathrm{fd}}^{*}$ : results from Cetin et al. [20].

Eqs. (9a)-(9d) can be non-dimensionalized with the following quantities,

$\Theta=\frac{T-T_{w}}{T_{i}-T_{w}}, B r=\frac{\mu u_{m}^{2}}{k\left(T_{i}-T_{w}\right)}, \widetilde{P e}=\frac{R e P r}{\rho_{s}\left(2-\rho_{s}^{2}\right)}$

$\xi=\rho_{s}^{2}\left(2-\rho_{s}^{2}\right) \frac{x}{P e R}, \eta=\frac{r}{R} \rho_{s}, u^{*}=\frac{u}{u_{m}}, \kappa=\frac{2-F_{t}}{F_{t}} \frac{2 \gamma}{\gamma+1} \frac{1}{\operatorname{Pr}}$.

Here, $\kappa$ is a parameter that represents the degree of temperature jump, defined from the temperature jump boundary condition, Eq. (9c). $\kappa=0$ corresponds to no temperature jump at the wall, while $\kappa=1.667$ is a typical value for air, which is the working fluid in many engineering applications and is taken so in this study. By introducing these non-dimensional parameters, energy equation and the boundary conditions become,

$\left(1-\eta^{2}\right) \frac{\partial \Theta}{\partial \xi}=\frac{1}{\eta} \frac{\partial}{\partial \eta}\left(\eta \frac{\partial \Theta}{\partial \eta}\right)+\frac{1}{\widetilde{P e}^{2}} \frac{\partial^{2} \Theta}{\partial \xi^{2}}+B r\left(\frac{\partial u^{*}}{\partial \eta}\right)^{2}$

$\eta=0, \frac{\partial \Theta}{\partial \eta}=0$

$\eta=\rho_{s}, \quad \Theta=C_{1} \rho_{s}\left(\frac{d \Theta}{d \eta}\right)_{\eta=\rho_{s}}$

where $C_{1}$ is defined as,

$C_{1}=-\frac{2-F_{t}}{F_{t}} \frac{2 \gamma}{\gamma+1} \frac{2 K n}{P r}=-2 K n \kappa$

$\xi=0, \Theta=1$
By using superposition, $\Theta$ can be decomposed as,

$\Theta=\Theta_{1}(\eta)+\Theta_{2}(\eta, \xi)$

where $\Theta_{1}(\eta)$ is the fully developed temperature profile and $\Theta_{2}(\eta, \xi)$ is the solution of the homogeneous equation. Once Eq. (12) is substituted into the energy equation, Eq. (11a), the resulting equation for $\Theta_{1}(\eta)$ becomes

$\frac{1}{\eta} \frac{\partial}{\partial \eta}\left(\eta \frac{\partial \Theta_{1}}{\partial \eta}\right)=-B r\left(\frac{\partial u^{*}}{\partial \eta}\right)^{2}$

$\Theta_{1}(\eta)$ can be derived by integrating Eq. (13) together with the symmetry at the centerline and the temperature-jump at the wall boundary conditions as,

$\Theta_{1}=\frac{-B r}{\left(2-\rho_{s}^{2}\right)^{2}}\left(\eta^{4}-\rho_{s}^{4}+4 \rho_{s}^{4} C_{1}\right)$

Then for the homogeneous part of the temperature distribution, $\Theta_{2}(\eta)$, the following equation together with the following boundary conditions should be solved.

$\left(1-\eta^{2}\right) \frac{\partial \Theta_{2}}{\partial \xi}=\frac{1}{\eta} \frac{\partial \Theta_{2}}{\partial \eta}+\frac{\partial^{2} \Theta_{2}}{\partial \eta^{2}}+\frac{1}{\widetilde{P e}^{2}} \frac{\partial^{2} \Theta_{2}}{\partial \xi^{2}}$

$\eta=0, \frac{\partial \Theta_{2}}{\partial \eta}=0$

$\eta=\rho_{s}, \quad \Theta_{2}=C_{1} \rho_{s}\left(\frac{d \Theta_{2}}{d \eta}\right)_{\eta=\rho_{s}}$

$\xi=0, \Theta_{2}=1-\Theta_{1}$.

It is assumed that the solution to the boundary value problem is of the form given below [23-26],

$\Theta_{2}=\sum_{n=1}^{\infty} A_{n} Y_{n}(\eta) \exp \left(-\lambda_{\mathrm{n}}^{2} \xi\right)$

where $A_{n}$ are the coefficients, $Y_{n}$ are the eigenfunctions, and $\lambda_{n}$ are the eigenvalues. Substituting Eq. (16) into Eq. (15a), the following non-

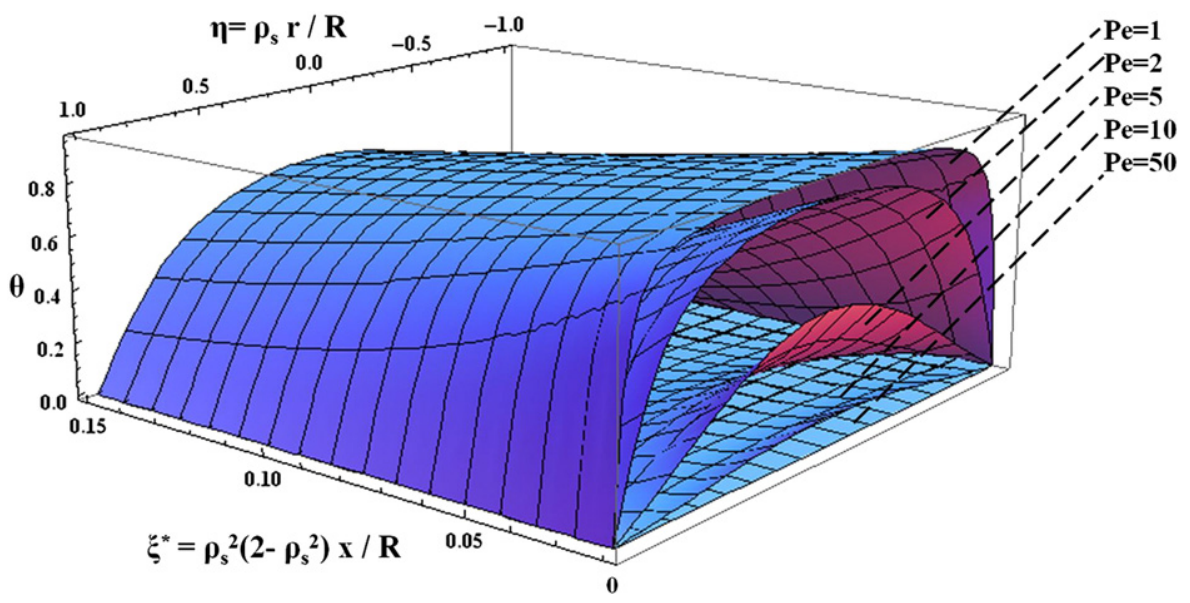

Fig. 2. Temperature profiles for different $\mathrm{Pe}$ with $\mathrm{Kn}=0, \mathrm{Br}=0$ and $\kappa=1.667$. 

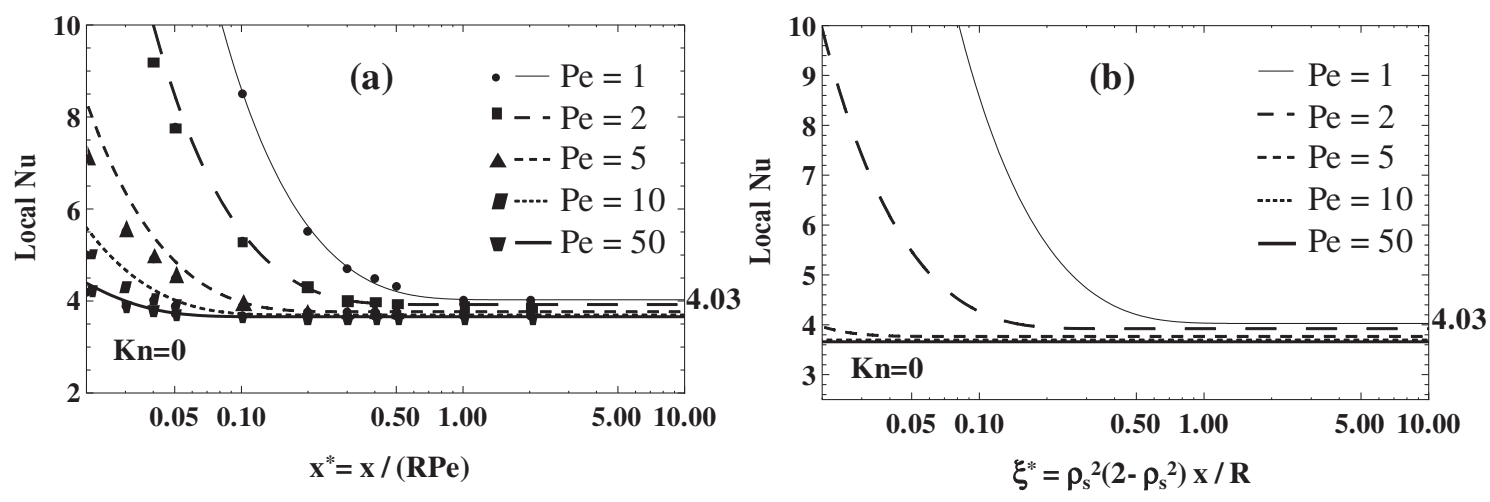

Fig. 3. Variation of local Nusselt number along (a) $x^{*}$ (with data points from Hennecke [30]) and (b) $\xi^{*}$, for different Peclet number values with Kn $=0$, Br $=0$ and $\kappa=1.667$.

linear problem can be obtained,

$\frac{d^{2} Y_{n}(\eta)}{d \eta^{2}}+\frac{1}{\eta} \frac{d Y_{n}(\eta)}{d \eta}+\lambda_{\mathrm{n}}^{2}\left(1-\eta^{2}+\frac{\lambda_{\mathrm{n}}^{2}}{P e^{2}}\right) Y_{n}(\eta)=0$

$\eta=0, \frac{d Y_{n}(\eta)}{d \eta}=0$

$\eta=\rho_{s}, \quad Y_{n}(\eta)=C_{1} \rho_{s}\left(\frac{d Y_{n}(\eta)}{d \eta}\right)_{\eta=\rho_{s}}$

Note that, when $C_{1}=0$ (i.e. $\rho_{s}=1$ ), and $\widetilde{\mathrm{Pe}}=\mathrm{Pe}$, the problem is equivalent to the macrotube problem [27]. Under the symmetric boundary condition, Eq. (17b), the solution of Eq. (17a) can be represented as,

$Y(\eta)=1 F 1(a, c ; z) \exp \left(-\lambda \frac{\eta^{2}}{2}\right)$

where $1 F 1(a, c ; z)$ is Kummer's confluent hypergeometric function and,

$a=\frac{1}{2}-\frac{\lambda}{4}-\frac{\lambda^{3}}{4 \widetilde{\mathrm{Pe}}^{2}}$

$c=1$

$z=\gamma^{2}=\lambda \eta^{2}$

Detailed information about hypergeometric functions can be found elsewhere [28].

The eigenvalues can be determined by using the wall boundary condition, and the summation constants can be evaluated by using the inlet boundary condition. Note that eigenfunctions $Y(\eta)$ are not mutually orthogonal (referring to the standard Sturm-Liouville problem), since the eigenvalues occur non-linearly. To determine coefficients $A_{n}$, Gram-Schmidt orthogonal procedure is used. Details of Gram-Schmidt orthogonal procedure can be found in ref. [23].

Finally, the temperature distribution Eq. (12) becomes,

$$
\begin{aligned}
\Theta= & \frac{-B r}{\left(2-\rho_{s}^{2}\right)^{2}}\left(\eta^{4}-\rho_{s}^{4}+4 \rho_{s}^{4} C_{1}\right) \\
& +\sum_{n} A_{n} 1 F 1\left(a, c ; \lambda_{n} \eta^{2}\right) \exp \left(-\lambda_{\mathrm{n}} \frac{\eta^{2}}{2}\right) \exp \left(-\lambda_{\mathrm{n}}{ }^{2} \xi\right) .
\end{aligned}
$$

Once the temperature distribution is determined, the local Nusselt number can be determined as follows,

$N u_{\xi}=\frac{h_{\xi} 2 R}{k}=-\frac{2}{\Theta_{m}} \rho_{s}\left(\frac{\partial \Theta}{\partial \eta}\right)_{\eta=\rho_{s}}$

where $\Theta_{m}$ is the non-dimensional temperature and defined as,

$\Theta_{m}(\xi)=\frac{1}{\rho_{s}^{2}} \int_{0}^{\rho_{s}} u^{*}(\eta) \Theta(\eta, \xi) \eta d \eta$.

In some of the results, classical dimensionless quantities given below are used for easier comparison with literature:

$x^{*}=\frac{x}{R P e}, \quad r^{*}=\frac{r}{R}$.

Furthermore, dimensionless axial direction, $\xi$, is transformed into the form below by excluding the term Pe to investigate the effect of Pe
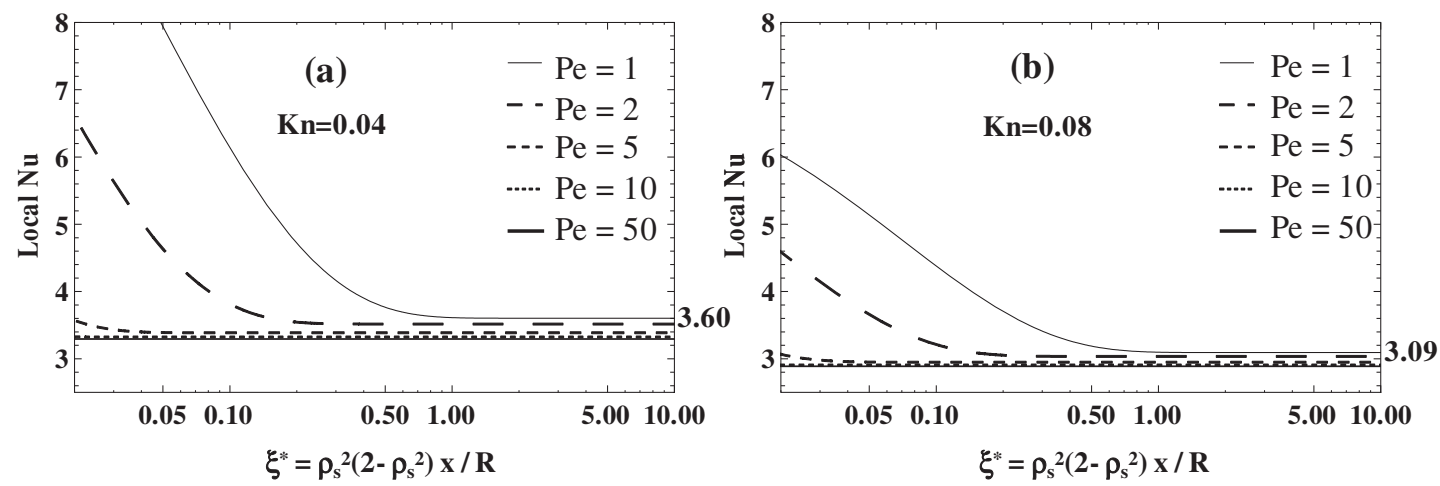

Fig. 4. Variation of local Nusselt number along $\xi^{*}$ for different Peclet number values at (a) $\mathrm{Kn}=0.04$ and $(\mathrm{b}) \mathrm{Kn}=0.08$, with $\mathrm{Br}=0$ and $\kappa=1.667$. 
onto axial conduction.

$\xi^{*}=\rho_{s}^{2}\left(2-\rho_{s}^{2}\right) \frac{x}{R}=\rho_{s}^{2}\left(2-\rho_{s}^{2}\right) x^{*} \mathrm{Pe}$

\section{Results and discussion}

The solution procedure is prepared with the help of Mathematica software. Eigenvalues are evaluated using the built in function root finder combined with a bracketing method. The method works up to a very high number of eigenvalues with high accuracy and short CPU times. However, for the orthogonalization part, the procedure may be complicated because of the oscillating characteristic of high order eigenfunctions. Integration of eigenfunctions results in excessive CPU time, which is not practical. This problem is eliminated by using Gaussian quadrature method for integrations. Gaussian integration with 100 points and 12 weights gives nearly the same result with direct integration in a relatively short time. The first 20 eigenvalues and corresponding coefficients for $\mathrm{Pe}=10,5$, and 1 are listed in Table 1 .

The fully developed $\mathrm{Nu}, \mathrm{Nu}_{\mathrm{fd}}$ values for the continuum case as a function of Pe are investigated. Just the first eigenvalues are enough to calculate $\mathrm{Nu}_{\mathrm{fd}}$, and are listed in Table 2 for a wide range of values for Pe. It can be seen that for $\mathrm{Pe}=10^{6}$, the well-known Nu value of 3.66 for laminar fully developed flow was determined and experienced no change for $\mathrm{Pe}=10^{9}$, which corresponds to $\mathrm{Pe}=\infty$ in the current study. $\mathrm{Nu}$ decreases from 4.18 at $\mathrm{Pe}=0$ to 3.66 for $\mathrm{Pe}=\infty$. Also, the solution shows the axial conduction effect up to $\mathrm{Pe}=10^{6}$. However, the results also suggest that the effect of axial conduction can be neglected for Pe values higher than 100 . A comparison of the present study with results from literature is given in Table 3 for $P e \leq 10$, where axial conduction seems to be more influential. The Table shows excellent agreement with the available solutions.

Slip flow regime is defined as the range of Kn between 0.001 and 0.1 . In slip-flow regime,two non-dimensional parameters, Kn and the parameter $\kappa$ affect the temperature field. Kn includes the effect of rarefaction and the parameter $\kappa$ includes the effect of gas and surface interaction, as given in Eq.(10). The fully developed $\mathrm{Nu}$ for different $\mathrm{Kn}$ together with the results from the literature are tabulated for different Pe in Table 4. Nu decreases with the increasing Kn as a result of the increase of temperature jump at the wall.

For the thermally developing region, accuracy of the solution mainly depends on the number of eigenvalues and eigenfunctions used in solution. Particularly as we get close to the entrance, more eigenfunctions are needed for an accurate calculation. However, a high number of eigenfunctions for a summation solution may still not be practical. Since functional microchannels have high length to diameter ratios, the resolution at the inlet does not play an important role for the overall picture. After several attempts, it is seen that solutions with 50,40 , and 30 eigenfunctions give the same results after $\xi^{*}=0.02$. As a result, 30 eigenfunctions are accepted as the suitable number for fairly good results for the thermally developing region.

Temperature profiles, through $\xi^{*}$ for different Pe, can be seen in Fig. 2. Increase of the thermal entrance length, $\mathrm{L}_{t}$, with a decrease in Pe can be visualized clearly. Decrease of Pe increases the axial conduction, which results in the rise of dimensionless temperature at any cross section and length required to achieve fully developed conditions.

Fig. $3 a$ and $b$ shows the effect of axial conduction through the thermal entrance region for $\mathrm{Kn}=0$. A minor difference with data points taken from Hennecke [30] shows the validation of the solution in Fig. 3-a. Similar to fully developed results, Nu increases with decreasing Pe. Furthermore, axial conduction effect is more influential at the beginning of the development region. As a result, it can be concluded that axial conduction is more important for the early part of thermal development region. Moreover thermal entrance length also increases with decreasing Pe.

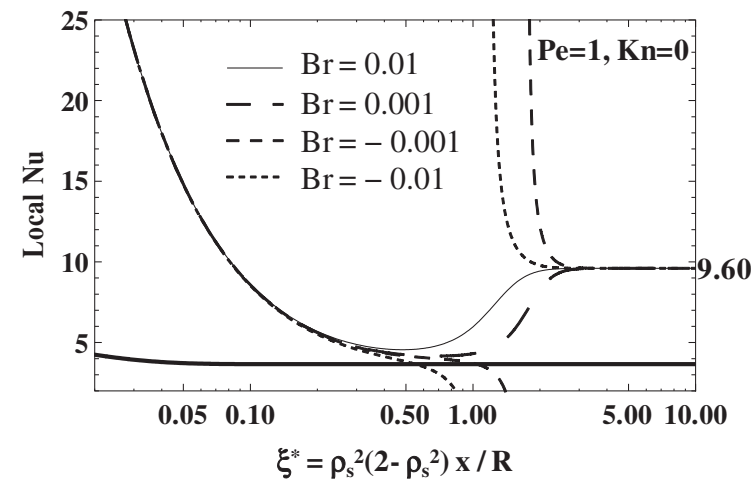

Fig. 5. Variation of local Nusselt number along $\xi^{*}$ for different Brinkman number values with $\mathrm{Pe}=1, \mathrm{Kn}=0$ and $\kappa=1.667$.

Fig. 4-a and b shows the variation of Nu for different Pe with Kn different from zero. Axial conduction for the slip flow case still has a high effect on $\mathrm{Nu}$ for different $\mathrm{Kn}$. However, its effect decreases as Kn increases. It can also be concluded that the effect of $\mathrm{Kn}$ is higher for low Pe values because of high axial conduction resulting in an increase in dimensionless temperature at any cross section, such as at the boundaries. In the slip flow regime, temperature gradients at the boundaries are the main influential factor for the temperature jump boundary condition. As a result, for low Pe values, temperature gradients and the resulting temperature jump at the pipe wall cause Kn to have a high effect on flow.

Fig. 5 shows the effect of positive and negative $\mathrm{Br}$ values for $\mathrm{Pe}=1$ and $\mathrm{Kn}=0$ case. First, Nu values are the same with no viscous dissipation case up to some portion of $\xi^{*}$ depending on the Br value. The main effect of viscous heating starts after that point and dominates the flow. $\mathrm{Nu}$ converges to the same value, 9.6, for all $\mathrm{Br}$ values different than 0 . As mentioned before, for positive $\mathrm{Br}$, which means fluid cooling, viscous dissipation enhances heat transfer. This can be seen from the sudden increase of Nu. Furthermore, the increase of Br results in the transfer of the jump-point towards the downstream direction similar to the increase of $\mathrm{L}_{\mathrm{t}}$, which is the main effect of the value of $\mathrm{Br}$. For negative $\mathrm{Br}$, which corresponds to fluid heating, Nu has a singularity where the bulk mean temperature of the fluid is equal to the wall temperature. Again, the value of $\mathrm{Br}$ alters the location of singularity as a result of the change in the amount of viscous dissipation. After the singularity, heat transfer changes direction as mentioned before.

Fig. 6 shows the axial conduction effect in the presence of viscous heating. It can be seen therein that axial conduction still has a high effect through the thermally developing region up to some value of $\xi^{*}$ depending on the Pe value. For Pe $=1$, axial conduction determines the local Nu up to $\xi^{*}=0.5$, after which viscous dissipation starts to influence the flow and dominates $\mathrm{Nu}_{\mathrm{fd}}$ as mentioned before. Before that location, Nu values are similar to no viscous dissipation

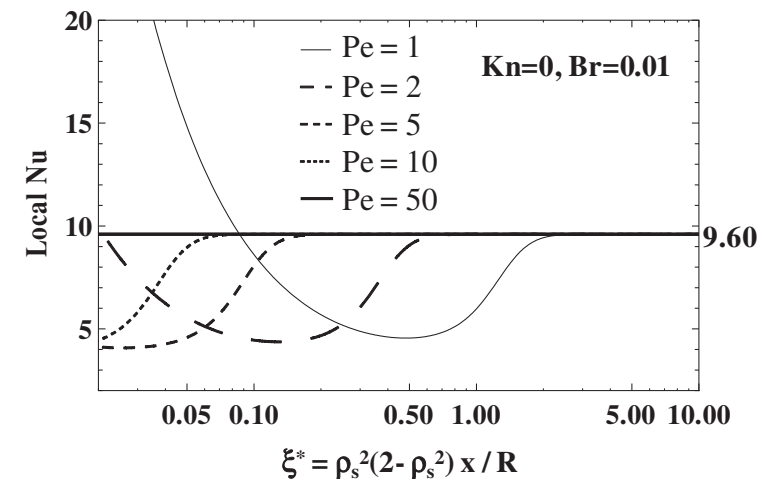

Fig. 6. Variation of local Nusselt number along $\xi^{*}$ for different Peclet number values with $\mathrm{Br}=0.01, \mathrm{Kn}=0$ and $\kappa=1.667$. 


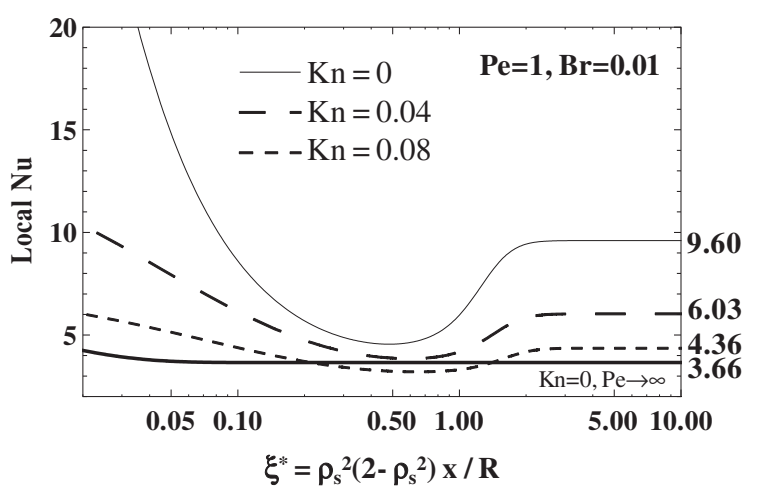

Fig. 7. Variation of local Nusselt number along $\xi^{*}$ for different Knudsen number values with $\mathrm{Pe}=1, \mathrm{Br}=0.01$ and $\kappa=1.667$.

case. Furthermore, the main impact of Pe is on the jump point location (sudden increase of $\mathrm{Nu}$ ) in the thermally developing region. Increase in Pe results in the movement of jump point towards downstream, similar to the influence of $\mathrm{Br}$ values, but its effect is more significant than the effect of Br.

Fig. 7 shows the effect of rarefaction for the positive $\mathrm{Br}$ case with $\mathrm{Kn}$ values different from zero. The dominant effect of viscous heating is reduced as a result of velocity slip boundary condition in micro flow, and increase in $\mathrm{Kn}$ decreases $\mathrm{Nu}_{\mathrm{fd}}$ values. Also, the increase in Kn results in the transition of jump point towards upstream, similar to $\mathrm{L}_{\mathrm{t}}$. Moreover, axial conduction effect for the slip flow regime is the same as continuum case in the presence of viscous dissipation.

Examination of the rarefaction affect in the case of viscous dissipation continues for negative $\mathrm{Br}$ value (fluid heating) in Fig. 8. Similar to the previous analysis, $\mathrm{Nu}_{\mathrm{fd}}$ values decrease with an increase in $\mathrm{Kn}$ and converge to the same values as the fluid cooling case (positive $\mathrm{Br}$ ) due to the change in the direction of heat transfer for $\mathrm{Br}=-0.01$. Furthermore, singular points move upstream as a result of increase in rarefaction.

\section{Summary and conclusions}

Steady state heat transfer for hydrodynamically developed, thermally developing microtube flow is studied by including the effects of axial conduction, viscous dissipation, and rarefaction. An analytical solution is obtained to increase the fundamental understanding of the physics of the problem. Orthogonal eigenfunctions are generated by the GramSchmidt orthogonalization procedure. Kummer's hypergeometric functions are used in the solution of the problem, and it is seen that the use of these functions are very effective by the help of the Mathematica

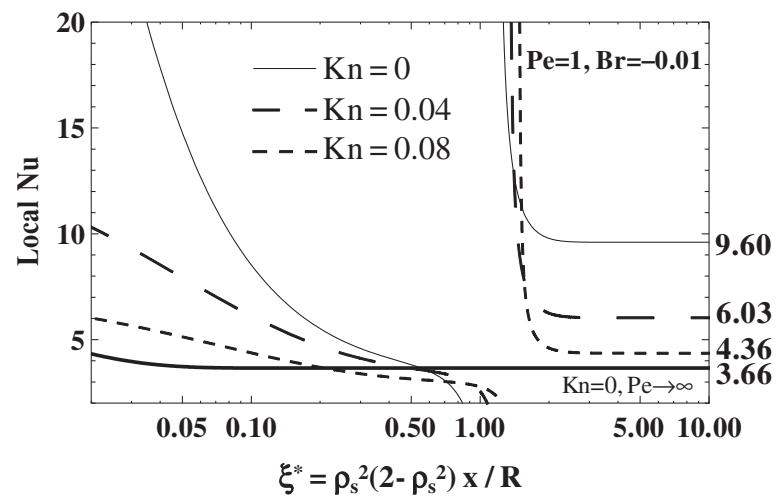

Fig. 8. Variation of local Nusselt number along $\xi^{*}$ for different Knudsen number values with $\mathrm{Pe}=1, \mathrm{Br}=-0.01$ and $\kappa=1.667$. software. Very good agreement is obtained with the available results in the literature.

The effects of four parameters; $\mathrm{Pe}, \mathrm{Kn}, \kappa$, and $\mathrm{Br}$, on the flow are discussed. Pe, varying between 1 and $\infty$, represents the dependency of the flow upon downstream conditions. Kn ranges from $\mathrm{Kn}=0$, which is the continuum case (i.e. flow in macro tubes), to $\mathrm{Kn}=0.1$, which is the upper limit of the slip flow regime. $\kappa$ parameter, representing temperature jump, is taken as $\kappa=1.667$, since it is the typical value for air, the working fluid for most of the engineering applications. $\mathrm{Br}$ is ranging from -0.01 , representing fluid heating, to 0 , which stands for the case without viscous dissipation, to 0.01 , which is for fluid cooling.

Results show that axial conduction has an important effect at low Pe and this effect increases with a decrease in Pe value. However, it is negligible for $\mathrm{Pe}>100$. The increase in axial conduction increases both $\mathrm{Nu}_{\mathrm{fd}}$ and local $\mathrm{Nu}$ values for all continuum (macro) and slip flow (micro), fully developed or thermally developing cases without viscous dissipation. Moreover, $\mathrm{L}_{\mathrm{t}}$ also increases as a result of increase in axial conduction. However, different from macro flow, the high effect of axial conduction for $\mathrm{Pe}<100$ decreases as a result of enhancement of rarefaction effect in micro flow because of high streamwise conduction.

In the presence of viscous dissipation, axial conduction has no effect on $\mathrm{Nu}_{\mathrm{fd}}$ but still affects the local Nu values up to some $\xi^{*}$ value depending on the Pe value. Before that point, viscous dissipation starts to influence the flow, local Nu values are similar with no viscous dissipation case. Again, similar to its effect on $\mathrm{L}_{t}$, the increase of axial conduction results in the movement of jump point (sudden increase of $\mathrm{Nu}$ ) towards upstream for $\mathrm{Br} \neq 0$ in both macro and micro cases.

For viscous dissipation, results show that $\mathrm{Nu}_{\mathrm{fd}}$ converges to 9.6 regardless of $\mathrm{Pe}$ or $\mathrm{Br}$ values for all conditions in the continuum case. Only through the thermally developing region, the effect of $\mathrm{Br}$ values can be visualized. For positive $\mathrm{Br}$, viscous heating enhances the heat transfer. However, negative values result in singular points, after which heat transfer changes direction. Moreover, the value of $\mathrm{Br}$ has an effect on the jump point; the increase of $\mathrm{Br}$ value results in the transition of the jump point towards downstream, similar to its effect on $L_{t}$, independent of its sign. All of the outcomes are also valid for the slip flow regime. However, different from macro flow, the dominant effect of viscous heating is reduced as a result of velocity slip boundary condition in micro flow; $\mathrm{Nu}_{\mathrm{fd}}$ values decrease with an increase in Kn. Moreover, $\mathrm{L}_{\mathrm{t}}$ also increases as a result of an increase in Kn, which also means transition of jump point towards upstream.

For all cases, the rarefaction effect, represented by the parameter $\kappa$ and $\mathrm{Kn}$, decreases the Nu values in slip flow regime when $\mathrm{Kn} \neq 0$. From this study, the following general conclusions can be obtained.

(1) Axial conduction should not be neglected for Pe $<100$ for all cases without viscous dissipation.

(2) In the presence of viscous dissipation, axial conduction should not be neglected for short pipes with $\mathrm{Pe}<100$.

(3) For viscous heating case, even for small $\mathrm{Br}$, fully developed $\mathrm{Nu}$ value experiences a jump in magnitude. The value of $\mathrm{Br}$ only affects the axial location of the jump. Therefore, the effect of viscous heating should be considered even for small $\mathrm{Br}$ with large length over diameter (L/D) ratios, which is the case for flows in micropipes.

(4) For a fixed $\kappa$ parameter, the deviation from continuum increases with increasing rarefaction and Nu values decrease with an increase in $\mathrm{Kn}$.

\section{Acknowledgments}

Financial support from the Turkish Scientific and Technical Research Council (TUBITAK), Grant No. 106M076, is greatly appreciated. 


\section{References}

[1] S. Kakac, Y. Yener, A. Pramuanjaroenkij, Convective Heat Transfer, CRC Press, Boca Raton, FL, 2014

[2] A. Beskok, G.E. Karniadakis, Simulation of heat and momentum transfer in complex micro-geometries, J. Thermophys. Heat Transf. 8 (1994) 355-370.

[3] G.A. Bird, Molecular Gas Dynamics and Direct Simulation of Gas Flows, Clarendon Press, Oxford, UK, 1994.

[4] M. Barisik, A. Beskok, Molecular free paths in nanoscale gas flows, Phys. Fluids Microfluid. Nanofluid. (2014), http://dx.doi.org/10.1007/s10404-014-1535-3.

[5] M. Barisik, A. Beskok, Scale effects in gas nano flows, Phys. Fluids 26 (2014) 052003.

[6] M. Barisik, A. Beskok, Surface-gas interaction effects on nanoscale gas flows, Microfluid. Nanofluid. 13 (5) (2012) 789-798.

[7] M. Barisik, A. Beskok, Molecular dynamics simulations of shear driven gas flows in nano-channels, Microfluid. Nanofluid. 11 (5) (2011) 611-622.

[8] M. Barisik, B. Kim, A. Beskok, Smart wall model for molecular dynamics simulations of nanoscale gas flows, Commun. Comput. Phys. 7 (2010) 977-993.

[9] M. Gad-el-Hak, The MEMS Handbook, CRC Press, New York, 2001.

[10] H.P. Kavehpour, M. Faghri, Y. Asako, Effects of compressibility and rarefaction on gaseous flows in microchannels, Numer. Heat Transfer, Part A 32 (1997) 677-696.

[11] E.B. Arkilic, K.S. Breuer, M.A. Schmidt, Gaseous flow in microchannels. Application of microfabrication to fluid mechanics, ASME FED. 197 (1994) 57-66.

[12] L. Graetz, Uber die warmeleitungsfahigkeit von flussigkeiten. Part 1 (On the thermal conductivity of liquids), Annu. Rev. Phys. Chem. 18 (1883) 79-94.

[13] W. Nusselt, Die abhangigkeit der warmeubergangszhal von der rohrlange (The dependence of the heat transfer coefficient on the tube length), VDI Z. 54 (1910) 1154-1158.

[14] W.M. Deen, Analysis of Transport Phenomena, Oxford University Press, Oxford, UK, 1998.

[15] W. Sun, S. Kakac, A.G. Yazicioglu, A numerical study of single-phase convective heat transfer in microtubes for slip flow, Int. J. Therm. Sci. 46 (11) (2007) 1084-1094.

[16] D.C. Knupp, R.M. Cotta, C.P. Naveira-Cotta, S. Kakaç, Transient conjugated heat transfer in microchannels: integral transforms with single domain formulation, Int. J. Therm. Sci. 88 (2015) 248-257.
[17] N.G. Hadjiconstantinou, O. Simek, Constant wall temperature Nusselt number in micro and nano-channels, J. Heat Transf. 124 (2002) 356-364.

[18] H.E. Jeong, J.T. Jeong, Extended Graetz problem including streamwise conduction and viscous dissipation in microchannels, Int. J. Heat Mass Transf. 49 (13-14) (2006) 2151-2157.

[19] B. Cetin, H. Yuncu, S. Kakac, Gaseous flow in microchannels with viscous dissipation, Int. J. Transp. Phenom. 8 (2006) 297-315

20] B. Cetin, A.G. Yazicioglu, S. Kakac, Fluid flow in microtubes with axial conduction including rarefaction and viscous dissipation, Int. Commun. Heat Mass 35 (2008) 535-544.

[21] B. Cetin, A.G. Yazıcıoğlu, S. Kakaç, Slip-flow heat transfer in microtubes with axia conduction and viscous dissipation-an extended Graetz problem, Int. J. Therm. Sci. 48 (2009) 1673-1678.

[22] B. Çetin, S. Zeinali, Analysis of heat transfer for a low Peclet number microtube flow using second-order slip model: an extended-Graetz problem, J. Eng. Math. 89 (1) (2014) 13-25.

[23] P. Dutta, K. Horiuchi, H.M. Yin, Thermal characteristics of mixed electroosmotic and pressure-driven microflows, Comput. Math. Appl. 52 (5) (2006) 651-670.

[24] K. Horiuchi, P. Dutta, A. Hossain, Joule-heating effects in mixed electroosmotic and pressure-driven microflows under constant wall heat flux, J. Eng. Math. 54 (2006) 159-180.

[25] F.E. Larrode, C. Housiadas, Y. Drossinos, Slip flow heat transfer in circular tubes, Int. J. Heat Mass Transf. 43 (2000) 2669-2680.

[26] W.M. Deen, Analysis of Transport Phenomena, Oxford University Press, New York 1998. 387 (Eq 9.5-7)

[27] J. Lahjomri, A. Oubarra, Analytical solution of the Graetz problem with axial conduction, J. Heat Transf. 121 (1999) 1078-1083 (Eq 10).

[28] G.B. Arkfen, H.J. Weber, Mathematical Methods for Physicists, Academic Press, New York, 2005

[29] R.K. Shah, A.L. London, Laminar Flow Forced Convection in Ducts, Academic Press, New York, 1978.

[30] D.K. Hennecke, Heat transfer by the Hagen-Poiseuille flow in the thermal development regime with axial conduction, Warme Stoffubertrag 1 (1968) 177-184. 